Artigo Revisão

\title{
Produção científica brasileira de enfermagem em terapia intensiva de 1995 a 2004
}

\author{
Brazilian researches on intensive care unit from 1995 to 2004 \\ Producción científica brasileña de enfermería en cuidados intensivos de 1995 al 2004

\begin{abstract}
Adriana Janzantte Ducci ${ }^{1}$, Daniella Vianna Correa Krokoscz ${ }^{1}$, Sheila Cristina Tosta Bento ${ }^{1}$, Kátia Grillo Padilha $^{2}$, Miako Kimura², Ana Maria Kazue Miyadahira ${ }^{3}$
\end{abstract}

\begin{abstract}
RESUMO
Objetivo: Investigar a produção científica de enfermagem em Unidade de Terapia Intensiva (UTI), no Brasil, no período de 1995 a 2004. Métodos: Foram pesquisados artigos divulgados em dez periódicos nacionais e analisados os dados referentes à freqüência de publicação, autoria, método de pesquisa utilizado, tema e população do estudo. Resultados: Do total de 3464 artigos publicados, 97 (2,8\%) eram específicos de UTI. Cerca de 38,0\% referiam-se a estudos de alunos de mestrado/doutorado, prevalecendo as pesquisas com abordagem qualitativa (49,5\%). Quanto ao tema, 23,7\% pesquisaram a atuação profissional dos trabalhadores envolvidos no cuidado, enquanto $21,6 \%$ abordaram os aspectos emocionais da assistência em UTI. Destaca-se o surgimento da temática iatrogenia como objeto de investigação. A equipe de enfermagem foi a população estudada em quase metade dos artigos publicados. Conclusão: A produção científica brasileira em UTI ainda é incipiente, mantendo-se estável nas duas últimas décadas.
\end{abstract}

Descritores: Publicações; Pesquisa em enfermagem; Comunicação e divulgação científica; Cuidados intensivos

\begin{abstract}
Objective: To investigate nursing research in critical care in Brazil from 1995 to 2004. Methods: Selected articles from ten Brazilian scientific journals were analyzed regarding frequency of publication, authorship, research methodology, topic, and study population. Results: Among 3464 published articles, only 97 (2.8\%) were related to critical care. A great part of these studies $(38.1 \%)$ was conducted by Master's or Doctoral graduate students. Almost a half of the studies (49.5\%) used qualitative design. Main topics studied consisted of the professional performance of worker involved on care (23.7\%) and emotional aspects of nursing care (21.6\%). Iatrogenic occurrences also emerge as an important topic. Nursing team was a common sample $(50.0 \%)$ of the studies. Conclusion: Nursing research in critical care in Brazil although incipient remains stable in the last two decades.
\end{abstract}

Keywords: Publications; Nursing research; Scientific communication and diffusion; Intensive care

\section{RESUMEN}

Objetivo: Investigar la producción científica de enfermería en la Unidad de Cuidados Intensivos (UCI), en el Brasil, entre los años 1995 y 2004. Métodos: Los artículos de diez periódicos nacionales fueron analizados referentes a la frecuencia de publicación, autoría, método de investigación, tema y población del estudio. Resultados: Del total de 3464 artículos, 97 (2,8\%) se refirieron a UCI; 38,1\% a estudios realizados por alumnos de post grado en maestría o doctorado. El abordaje cualitativo (49,5\%) prevaleció. En cuanto a los temas, el 23,7\% se interesaron por investigar la actuación profesional de los trabajadores de la UCI. Los aspectos emocionales de la asistencia de enfermería fue el tema abordado en el 21,6\% de los trabajos. Se destaca el surgimiento del tema yatrogenia como objeto de investigación. El equipo de enfermería fue la población estudiada en cerca del 50,0\% de los artículos. Conclusion: La producción científica brasileña en la UCI es aún incipiente, manteniéndose estable en las últimas dos décadas.

Descriptores: Publicaciones; Investigación en enfermería; Comunicación y divulgación científica; Cuidados Intensivos

\footnotetext{
${ }^{1}$ Especialista em Terapia Intensiva. Integrantes do Grupo de Pesquisa Enfermagem em Unidade de Terapia Intensiva da Escola de Enfermagem da Universidade de São Paulo - USP - São Paulo (SP), Brasil.

${ }^{2}$ Professor Associado do Departamento de Enfermagem Médico-Cirúrgica da Escola de Enfermagem da Universidade de São Paulo - USP - São Paulo (SP), Brasil.

${ }^{3}$ Professor Titular do Departamento de Enfermagem Médico-Cirúrgica da Escola de Enfermagem da Universidade de São Paulo - USP - São Paulo (SP), Brasil.
} 


\section{INTRODUÇÃO}

O conhecimento produzido nas diversas áreas da enfermagem vem crescendo gradativamente no Brasil, impulsionado pela criação dos cursos de pós-graduação stricto sensu na década de 70, após a reforma universitária ${ }^{(1-2)}$.

Os primeiros cursos de pós-graduação em enfermagem tiveram início na Escola de Enfermagem Anna Nery e na Escola de Enfermagem da Universidade de São Paulo em 1972 e 1973, respectivamente ${ }^{(1)}$. Tratando-se especificamente da enfermagem em Unidade de Terapia Intensiva (UTI), a produção científica é muito recente, uma vez que essa área de atuação profissional teve início na década de $70 \mathrm{com}$ a implantação das primeiras UTIs no Brasi ${ }^{(1,3)}$.

Estudo brasileiro ${ }^{(3)}$ que analisou comparativamente o conteúdo das pesquisas de enfermagem brasileiras e americanas em terapia intensiva e nas demais unidades médico-cirúrgicas, no período de 1975 a 1984, constatou a quase inexistência de pesquisas em UTI, apesar do aumento quantitativo da produção científica em enfermagem com pesquisas tanto descritivas quanto experimentais.

Dez anos depois, outro grupo de pesquisadoras deu seguimento a esse trabalho por meio de um estudo sistemático da produção científica de enfermagem em UTI no Brasil, compreendendo o período de 1985 a 1994, destacando que apenas $2,7 \%$ da produção científica nacional de enfermagem referiam-se à assistência intensiva ${ }^{(4)}$.

Constatando uma lacuna referente aos últimos dez anos e considerando a importância de se obter dados sobre a produção de enfermagem em UTI, no Brasil, optou-se por realizar o presente estudo. Assim, foi estabelecido como objetivo analisar a situação atual da produção científica nessa área, por meio da identificação da freqüência de publicação, autoria, métodos de pesquisa utilizados tema e população do estudo. Pretende-se com este estudo, oferecer subsídios que direcionem o desenvolvimento de pesquisas por enfermeiros interessados na área de terapia intensiva.

\section{MÉTODO}

Trata-se de um estudo de levantamento bibliográfico, que teve como foco os artigos científicos da área de enfermagem em terapia intensiva, publicados em periódicos nacionais de enfermagem, no período de 1995 a 2004.

Foram selecionados dez periódicos de classificação internacional B e C e nacional B considerados como representativos dos principais Estados brasileiros.

Os dados foram obtidos pelas autoras, por meio de busca ativa nos periódicos classificados em 2005 pelo
Índice Qualis da Coordenação de Aperfeiçoamento de Pessoal de Ensino Superior (CAPES) $)^{(5)}$, como descritos a seguir: Revista Latino-americana de Enfermagem (Internacional B), Acta Paulista de Enfermagem, Revista da Escola de Enfermagem da USP, Texto \& Contexto Enfermagem, Revista Gaúcha de Enfermagem, Revista Brasileira de Enfermagem (Internacional C), Revista Mineira de Enfermagem, Revista Paulista de Enfermagem, Revista de Enfermagem da UERJ e Revista Baiana de Enfermagem (Nacional B).

Os artigos específicos de UTI deveriam conter no título do trabalho um dos seguintes descritores: Unidade de Terapia Intensiva, Centro de Terapia Intensiva, Paciente Crítico, Cuidado Intensivo.

Foram incluídos neste estudo, apenas os artigos classificados como de pesquisa e de revisão de literatura, sendo excluídos os editoriais, artigos de reflexão, relatos de experiência, ensaios, documentários, notas prévias, resumos de teses e resenhas, além de números de edições especiais comemorativas.

Após a localização dos periódicos, a coleta de dados foi realizada por meio de um instrumento criado para este fim, contendo: título do periódico e do artigo, autoria (número de autores e respectiva categoria), método de pesquisa utilizado (quantitativo, qualitativo, qualitativo/ quantitativo), tema e população/objeto de estudo.

Para a análise dos artigos segundo autoria, levou-se em consideração a categoria do primeiro autor, sendo assim denominada: docente, enfermeira assistencial, aluno de graduação, de especialização e de pós-graduação stricto sensu.

Quanto à abordagem metodológica, as pesquisas foram classificadas como quantitativas, qualitativas, quanti/ qualitativas ou de revisão de literatura.

A coleta de dados foi efetuada com base nas informações contidas nos resumos e na leitura do artigo na íntegra.

Os dados foram armazenados em um banco de dados eletrônico, sendo os resultados analisados segundo valores absolutos e percentuais e apresentados em forma de tabelas.

\section{RESULTADOS}

A Tabela 1, a seguir, mostra a distribuição das publicações em cada um dos periódicos, segundo o assunto abordado (gerais ou de UTI).

A Tabela 1 mostra que, no período de 1995 a 2004, foram publicados 3464 artigos nos dez periódicos analisados. Desse total, apenas $97(2,8 \%)$ referiam-se especificamente à assistência intensiva.

Observa-se que as revistas da Escola de Enfermagem da USP $(24,8 \%)$, Latino-americana de Enfermagem (16,5\%), Brasileira de Enfermagem (13,4\%), Acta Paulista 
de Enfermagem (10,3\%) e Paulista de Enfermagem $(10,3 \%)$ foram as que mais publicaram artigos específicos de terapia intensiva, quando comparadas com as demais revistas.

A seguir serão analisadas com maior detalhamento as publicações específicas de enfermagem em UTI identificadas na década de 1995 a 2004

Tabela 1 - Artigos publicados em periódicos nacionais de Enfermagem, no período de 1995 a 2004, segundo periódicos e assuntos. São Paulo, 2005.

\begin{tabular}{lrrrr}
\hline \multirow{2}{*}{ Periódicos } & \multicolumn{5}{c}{ Assuntos } \\
\cline { 2 - 6 } & \multicolumn{2}{c}{ Gerais } & \multicolumn{2}{c}{ UTI } \\
\cline { 2 - 5 } & $\mathrm{n}$ & $\%$ & $\mathrm{n}$ & $\%$ \\
\hline Rev Esc Enfermagem USP & 455 & 13,1 & 24 & 24,8 \\
Rev Latinoam Enfermagem & 712 & 20,6 & 16 & 16,5 \\
Rev Bras Enfermagem & 651 & 18,8 & 13 & 13,4 \\
Acta Paul Enfermagem & 359 & 10,4 & 10 & 10,3 \\
Rev Paul Enfermagem & 152 & 4,4 & 10 & 10,3 \\
Texto \& Contexto & 469 & 13,5 & 8 & 8,3 \\
Enfermagem & 160 & 4,6 & 8 & 8,3 \\
Rev Gaúch Enferm & 257 & 7,4 & 4 & 4,1 \\
Rev Enfermagem UERJ & 155 & 4,5 & 2 & 2,0 \\
REME Rev Min Enferm & 94 & 2,7 & 2 & 2,0 \\
Rev Baiana Enfermagem & 3.464 & 100,0 & 97 & 100,0 \\
\hline Total & & &
\end{tabular}

Tabela 2 - Publicações de enfermagem em terapia intensiva em periódicos nacionais nos períodos de 19951999 e 2000-2004, segundo categorias dos autores. São Paulo, 2005.

\begin{tabular}{lrrrrrrr}
\hline \multirow{2}{*}{ Categorias } & \multicolumn{9}{c}{ Ano de publicação } & & \\
\cline { 2 - 5 } & \multicolumn{2}{c}{$1995-$} & \multicolumn{2}{c}{$2000-$} & & \\
& \multicolumn{2}{c}{1999} & \multicolumn{2}{c}{2004} & & \\
\cline { 2 - 5 } & $\mathrm{n}$ & $\%$ & $\mathrm{n}$ & $\%$ & $\mathrm{n}$ & $\%$ \\
\hline Aluno de pós-graduação & 10 & 34,5 & 27 & 39,7 & 37 & 38,1 \\
Docente & 9 & 31,0 & 15 & 22,1 & 24 & 24,7 \\
Aluno de graduação & 6 & 20,7 & 9 & 13,2 & 15 & 15,5 \\
Aluno de especialização & 3 & 10,3 & 10 & 14,7 & 13 & 13,4 \\
Enfermeira assistencial & 1 & 3,5 & 7 & 10,3 & 8 & 8,3 \\
\hline Total & 29 & 100,0 & 68 & 100,0 & 97 & 100,0 \\
\hline
\end{tabular}

A Tabela 2 mostra um expressivo aumento de publicações no segundo qüinqüênio analisado (20002004), sendo nele identificados 68 artigos, que correspondem a $70,1 \%$ do total publicado.
Quanto à categoria dos autores, 38,1\% dos artigos foram publicados por alunos de programas de pósgraduação de mestrado ou doutorado, enquanto que, aproximadamente, $25,0 \%$ dos artigos foram publicados por docentes vinculados a instituições de ensino.

Tabela 3 - Publicações de enfermagem em terapia intensiva em periódicos nacionais, nos períodos de 19951999 e 2000-2004, segundo métodos de pesquisa utilizados. São Paulo, 2005.

\begin{tabular}{lrrrrrrr} 
& \multicolumn{4}{c}{ Ano de publicação } & \multirow{2}{*}{ Total } \\
\cline { 2 - 5 } Métodos de pesquisa & \multicolumn{3}{c}{$1995-$} & $2000-$ & & \\
& \multicolumn{2}{c}{1999} & 2004 & & \\
\cline { 2 - 5 } & $\mathrm{n}$ & $\%$ & $\mathrm{n}$ & $\%$ & $\mathrm{n}$ & $\%$ \\
\hline Qualitativo & 14 & 48,3 & 34 & 50,0 & 48 & 49,5 \\
Quantitativo & 13 & 44,8 & 34 & 50,0 & 47 & 48,5 \\
Quantitativo/qualitativo & 2 & 6,9 & - & - & 2 & 2,0 \\
\hline Total & 29 & 100,0 & 68 & 100,0 & 97 & 100,0 \\
\hline
\end{tabular}

Os dados da Tabela 3 revelam uma distribuição eqüitativa entre as pesquisas com abordagem qualitativa $(49,5 \%)$ e quantitativa (48,5\%), com discreto predomínio dos métodos qualitativos nos dois períodos analisados.

Pode-se verificar pelos dados da Tabela 4 que, no geral, a atuação profissional $(23,7 \%)$ e os aspectos emocionais da assistência de enfermagem $(21,6 \%)$ foram os temas mais explorados pelos pesquisadores. No entanto, comparando-se os qüinqüênios analisados, notase que houve um incremento no percentual de pesquisas sobre aspectos emocionais da assistência de enfermagem e iatrogenia e diminuição proporcional de estudos sobre atuação profissional e necessidades e problemas de familiares. Observa-se, também, que temas relativos à saúde ocupacional, à caracterização de pacientes e a procedimentos de enfermagem e terapêuticos, não abordados no primeiro qüinqüênio, passaram a ser investigados no seguinte, embora em baixas freqüências.

Quanto à população/objeto de estudo, observa-se na Tabela 5 que a equipe de enfermagem foi o foco de interesse em 50,5\% das pesquisas analisadas, seguida pelos pacientes e familiares, embora em porcentagens expressivamente menores, respectivamente, 19,6\% e $14,4 \%$. No entanto, a análise por qüinqüênio mostra aumento proporcional de artigos com essas populações.

\section{DISCUSSÃO}

Analisando a Tabela 1, observou-se que a produção científica de enfermagem em terapia intensiva, no período de 1995 a 2004, foi principalmente veiculada nos periódicos Revista da Escola de Enfermagem da 
USP, Revista Latino-americana de Enfermagem e Revista Brasileira de Enfermagem, todos com classificação Internacional pelo Índice Qualis da CAPES. Esse resultado era, de certo modo esperado, pelo menos em relação aos dois últimos periódicos, dada a sua maior periodicidade e número de edições anuais.

Tabela 4 - Publicações de enfermagem em terapia intensiva em periódicos nacionais, nos períodos de 19951999 e 2000-2004, segundo temas abordados. São Paulo, 2005.

\begin{tabular}{|c|c|c|c|c|c|c|}
\hline \multirow{3}{*}{ Temas abordados } & \multicolumn{4}{|c|}{ Ano de publicação } & \multirow{2}{*}{\multicolumn{2}{|c|}{ Total }} \\
\hline & \multicolumn{2}{|c|}{$\begin{array}{l}1995- \\
1999\end{array}$} & \multicolumn{2}{|c|}{$\begin{array}{l}2000- \\
2004\end{array}$} & & \\
\hline & $\mathrm{n}$ & $\%$ & $\mathrm{n}$ & $\%$ & $\mathrm{n}$ & $\%$ \\
\hline Atuação profissional & 9 & 31 & 14 & 20,6 & 23 & 23,7 \\
\hline $\begin{array}{l}\text { Aspectos emocionais da } \\
\text { assistência de } \\
\text { enfermagem }\end{array}$ & 4 & 13,8 & 17 & 25,0 & 21 & 21,6 \\
\hline Iatrogenia & 1 & 3,5 & 9 & 13,3 & 10 & 10,3 \\
\hline $\begin{array}{l}\text { Necessidades e } \\
\text { problemas de familiares }\end{array}$ & 3 & 10,3 & 6 & 8,9 & 9 & 9,3 \\
\hline $\begin{array}{l}\text { Necessidades e } \\
\text { problemas de pacientes }\end{array}$ & 1 & 3,5 & 3 & 4,4 & 4 & 4,1 \\
\hline $\begin{array}{l}\text { Metodologia da } \\
\text { assistência }\end{array}$ & 2 & 6,9 & 2 & 2,9 & 4 & 4,1 \\
\hline $\begin{array}{l}\text { Validação de } \\
\text { instrumentos de medida }\end{array}$ & 2 & 6,9 & 2 & 2,9 & 4 & 4,1 \\
\hline Saúde ocupacional & - & - & 4 & 5,9 & 4 & 4,1 \\
\hline $\begin{array}{l}\text { Caracterização do } \\
\text { ambiente de UTI }\end{array}$ & 1 & 3,5 & 2 & 2,9 & 3 & 3,1 \\
\hline $\begin{array}{l}\text { Perfil das pesquisas em } \\
\text { enfermagem }\end{array}$ & 1 & 3,5 & 2 & 2,9 & 3 & 3,1 \\
\hline Ensino-aprendizagem & 1 & 3,5 & 1 & 1,5 & 2 & 2,1 \\
\hline $\begin{array}{l}\text { Procedimentos de } \\
\text { enfermagem e } \\
\text { terapêticos }\end{array}$ & - & - & 2 & 2,9 & 2 & 2,1 \\
\hline $\begin{array}{l}\text { Caracterização de } \\
\text { pacientes }\end{array}$ & - & - & 2 & 2,9 & 2 & 2,1 \\
\hline $\begin{array}{l}\text { Diagnósticos de } \\
\text { enfermagem }\end{array}$ & 1 & 3,5 & 1 & 1,5 & 2 & 2,1 \\
\hline Outros* & 3 & 10,3 & 1 & 1,5 & 4 & 4,1 \\
\hline Total & 29 & 00,0 & 68 & 100,0 & 97 & 100,0 \\
\hline
\end{tabular}

* Custos em UTI, avaliação da tecnologia, qualidade de vida e caracterização da equipe de enfermagem.

À semelhança de estudo realizado dez anos atrás(4), houve predomínio de publicações em revistas editadas no Estado de São Paulo, provavelmente devido a maior concentração de UTIs na região Sudeste do país, no Estado e no Município de São Paulo.

Ao se comparar os resultados desta investigação com estudo que realizou análise retrospectiva da produção científica nacional de enfermagem em UTI, no período de 1985 a 1994, ${ }^{(4)}$ observou-se que, em números absolutos, houve um aumento no número de publicações, de 45 para 97, nessa última década. No entanto, ao se comparar a produção científica em UTI com o total de publicações no período, verificou-se que as pesquisas nesta área ainda continuam escassas, representando apenas $2,7 \%$ do total de publicações no período de 1985 a 1994 e 2,8\%, de 1995 a 2004.

Tabela 5 - Publicações de enfermagem em terapia intensiva em periódicos nacionais nos períodos de 19951999 e 2000-2004, segundo população/objeto de estudo. São Paulo, 2005.

\begin{tabular}{|c|c|c|c|c|c|c|}
\hline \multirow{3}{*}{$\begin{array}{l}\text { População/objeto de } \\
\text { estudo }\end{array}$} & \multicolumn{4}{|c|}{ Ano de publicação } & \multicolumn{2}{|c|}{ Total } \\
\hline & \multicolumn{2}{|c|}{$\begin{array}{l}1995- \\
1999 \\
\end{array}$} & \multicolumn{2}{|c|}{$\begin{array}{c}2000- \\
2004 \\
\end{array}$} & \multirow[b]{2}{*}{$\mathrm{n}$} & \multirow[b]{2}{*}{$\%$} \\
\hline & $\mathrm{n}$ & $\%$ & $\mathrm{n}$ & $\%$ & & \\
\hline Equipe de enfermagem & 17 & 58,6 & 32 & 47,1 & 49 & 50,5 \\
\hline Pacientes internados & 5 & 17,2 & 14 & 20,6 & 19 & 19,6 \\
\hline Familiares & 4 & 13,8 & 10 & 14,7 & 14 & 14,4 \\
\hline Ambiente de UTI & 2 & 6,9 & 5 & 7,3 & 7 & 7,2 \\
\hline Pesquisas & 1 & 3,5 & 4 & 5,9 & 5 & 5,2 \\
\hline Equipe multiprofissional & - & - & 3 & 4,4 & 3 & 3,1 \\
\hline Total & 29 & 100,0 & 68 & 100,0 & 97 & 100,0 \\
\hline
\end{tabular}

Podem justificar os resultados encontrados, a diversidade de áreas a serem exploradas na enfermagem, aliadas ao pouco interesse dos profissionais e pesquisadores em estudar aspectos relacionados a este ambiente, resultando na escassa produção científica em UTI na década em questão. No entanto, ao se considerar a terapia intensiva como um ambiente que requer contínuo aperfeiçoamento, para o que contribuem as publicações científicas, tal constatação é preocupante, principalmente quando se leva em conta que, comparativamente à produção da década anterior, não houve crescimento significativo.

Os resultados apresentados na Tabela 2 indicam que, no Brasil, a produção científica dessa área ainda é fundamentalmente produzida por alunos de Programas de Pós-Graduação stricto sensu, fato que se pode justificar pela valorização da produção discente nesses programas.

Sobre a menor participação dos docentes como primeiro autor, há que se considerar que grande parte da produção do docente é vinculada à orientação de teses, dissertações ou trabalhos de disciplinas, resultando em publicações cuja principal autoria é dos alunos orientados.

Em relação aos alunos de graduação, tem se observado, nos últimos anos, uma inserção cada vez mais precoce em projetos de iniciação científica estimulada pelos cursos de graduação e pelas agências financiadoras que têm, como meta, o desenvolvimento 
de uma massa crítica de jovens pesquisadores no país.

Apesar disso, neste estudo, a publicação de trabalhos por parte dos alunos graduandos em número menos expressivo $(15,5 \%)$ e, ainda, com diminuição do percentual no último qüinqüênio, pode ser atribuída tanto à falta de investimento no envio de trabalhos já finalizados para publicação, desmotivação no processo, geralmente longo, necessidades de ajustes do texto para publicação, como também à mudança de prioridades ao final do curso de graduação.

Outro problema que ainda se observa entre alunos de graduação é a falta de conhecimento mais consolidado sobre metodologia de pesquisa, resultando em pouca habilidade para o desenvolvimento e consumo de pesquisas $^{(1)}$.

Referente à participação dos enfermeiros assistenciais na produção de conhecimento em cuidados intensivos, proporções ainda pequenas de publicações por alunos dos cursos de especialização $(13,4 \%)$ e enfermeiros assistenciais $(8,3 \%)$ foram encontradas. A análise por qüinqüênio, porém, mostra resultados satisfatórios, com um aumento de cerca de $15,0 \%$ na participação desses profissionais. Apesar desses achados, os resultados apontam para a necessidade de incentivo e apoio contínuos por parte de professores e dirigentes hospitalares para que seja apoiada a realização de pesquisas que resultem em publicações na área da assistência intensiva.

Os cursos de especialização têm sido apontados por alguns autores ${ }^{(2,6-8)}$ como cursos de fundamental importância à estimulação das atividades de pesquisa em campo por meio de sua interação com as funções assistenciais, o que contribui para a qualidade da assistência, maior satisfação do profissional e reconhecimento do trabalho realizado.

Nesse sentido, estudo realizado no período de 1989 a 1993, constatou que 91,7\% dos hospitais, cujos profissionais de enfermagem realizaram ou participaram de pesquisas, aplicaram os resultados dos estudos na própria instituição ${ }^{(8)}$. No entanto, esse mesmo estudo mostrou que, das 154 pesquisas realizadas em instituições públicas e particulares, apenas $60(38,9 \%)$ foram publicadas.

Pode-se constatar que, se a publicação não se efetiva, o trabalho não é divulgado e seus resultados ficam restritos aos profissionais que o desenvolveram e que deles têm conhecimento. Assim, considerando que a divulgação é parte final do processo de produção científica, investimentos para que os enfermeiros divulguem os seus estudos devem ser continuamente enfatizados.

Conforme dados da Tabela 3, verificou-se que, quanto ao método de investigação, pesquisas com abordagem qualitativa $(49,5 \%)$ e quantitativa $(48,5 \%)$ foram equivalentes, com discreto predomínio dos métodos qualitativos. Tais dados contrapõem-se aos encontrados nos estudos retrospectivos realizados nos períodos de 1975 a $1984^{(3)}$ e de 1985 a $1994^{(4)}$ em que, respectivamente, $99,2 \%$ e $98,0 \%$ das pesquisas utilizaram a abordagem quantitativa.

Nesse sentido, é possível que a maior utilização do método qualitativo para o estudo de diferentes fenômenos na enfermagem tenha ocorrido não só pela natureza dos temas investigados, como também pelo maior domínio dos pesquisadores no uso das ferramentas qualitativas, abordagem que passou a ser inserida nos Programas de Pós-Graduação, após a década de 80 .

A temática investigada pelos pesquisadores, comparativamente à de décadas anteriores, mostrou algumas mudanças. Em meados das décadas de 70 e 80 (1975 a 1984), havia um predomínio de pesquisas descritivas e referentes à área assistencial, particularmente com enfoque nos aspectos biológicos. Os assuntos abordados com mais freqüência foram relacionados a procedimentos de enfermagem e terapêuticos $(25,2 \%)^{(3)}$. $\mathrm{Na}$ década subseqüente, ou seja, de 1985 a 1995, assuntos como necessidades e problemas do paciente $(24,4 \%)$ e metodologia da assistência $(15,0 \%)$ foram os mais explorados pelos pesquisadores ${ }^{(9)}$.

No presente estudo, na década analisada, encontrouse maior freqüência de investigações relacionados à atuação profissional dos enfermeiros (23,7\%), assim como, aspectos emocionais da assistência de enfermagem (21,6\%). Representando aproximadamente 11,0\% dos trabalhos publicados no período, destaca-se o surgimento da temática eventos adversos em UTI, possivelmente em função dos movimentos de Acreditação Hospitalar e busca por indicadores de avaliação de resultados.

$\mathrm{Na}$ análise por qüinqüênio, foi interessante observar que temáticas que não haviam sido abordadas anteriormente, como saúde ocupacional e caracterização do paciente crítico, assim como procedimentos de enfermagem e terapêuticos, abordados apenas nas décadas de 70 e 80, constituíram objeto de investigação dos pesquisadores, ainda que em baixas freqüências. Por outro lado, temas como: necessidades e problemas de familiares, metodologia de assistência, perfil das pesquisas em enfermagem, validação de instrumentos de medida, diagnóstico de enfermagem, caracterização do ambiente de UTI e processo ensino-aprendizagem tiveram proporções decrescentes de publicações nos últimos cinco anos estudados.

Em investigação que analisou as dissertações e teses nacionais realizadas na área de enfermagem em terapia intensiva, publicados nos catálogos do Centro de Estudos e Pesquisa em Enfermagem ${ }^{(10)}$, no período de 1979 a 1997, a prática do enfermeiro mostrou-se como o tema de pesquisa mais explorado. Sob esse enfoque, foram abordadas questões referentes à prática cotidiana, numa 
busca de apreensão do trabalho do enfermeiro, com estudos sobre competências, relações de poder, disputas e conflitos, sentimentos e percepções em relação ao seu trabalho, entre outros.

Também pesquisa que analisou a temática das monografias oriundas do Curso de Especialização em Enfermagem Intensiva da Escola de Enfermagem da Universidade Federal da Bahia ${ }^{(7)}$ encontrou predominância de artigos sobre a atuação da enfermeira em procedimentos de alta complexidade $(35,3 \%)$, seguida da organização/gerência do cuidar (21,6\%), produção/ processo de trabalho $(19,6 \%)$ e humanização da assistência $(17,6 \%)$. Constata-se, pois, que nos estudos mencionados, tal como ocorreu nesta investigação, a atuação profissional foi o principal foco de investigação, embora com sinais de declínio nos últimos cinco anos analisados.

A escassez de estudos sobre procedimentos técnicos e terapêuticos relativos ao cuidado de enfermagem em UTI, evidenciada neste estudo, em que apenas duas pesquisas foram publicadas, também foi citada em estudos anteriores $^{(3-4)}$. Tais dados demonstram, portanto, a necessidade de suprir essa lacuna com o desenvolvimento de estudos clínicos que possam contribuir, sobretudo, para uma prática de enfermagem baseada em evidências científicas.

No presente estudo, a equipe de enfermagem foi a população estudada em 50,5\% das investigações, sendo a sua atuação profissional a principal temática abordada. Pacientes e familiares constituíram a população do estudo em 34,0\% das publicações, com temas voltados aos aspectos emocionais da assistência intensiva.

\section{CONCLUSÃO}

A análise da produção científica de enfermagem em UTI, em dez periódicos nacionais de enfermagem, no período de 1995 a 2004, permitiu concluir que:

- O volume de publicações de enfermagem em UTI manteve-se em torno de $3 \%$ da produção científica nacional de enfermagem.

- Os periódicos nacionais que publicaram maior número de artigos com pesquisas relacionadas à enfermagem em terapia intensiva foram: Revista da Escola de Enfermagem da USP, Revista Latino-americana de Enfermagem e Revista Brasileira de Enfermagem, com $24,8 \%, 16,5 \%$ e $13,4 \%$ dos estudos, respectivamente.

- As pesquisas foram produzidas principalmente por alunos de pós-graduação stricto sensu $(38,1 \%)$ e docentes vinculados a esses programas $(24,7 \%)$.

- Houve distribuição eqüitativa entre as pesquisas com abordagem qualitativa $(49,5 \%)$ e quantitativa (48,5\%).

- Os temas de investigação foram predominantemente relacionados à exploração ou descrição da atuação profissional da equipe de enfermagem $(23,7 \%)$ e dos aspectos emocionais da assistência (21,6\%), sendo escassas as pesquisas sobre procedimentos técnicos e terapêuticos de enfermagem.

- A equipe de enfermagem foi a população estudada em 50,5\% dos trabalhos, seguida dos pacientes e familiares em menores proporções, ou seja, em 19,6\% e $14,4 \%$, respectivamente.

\section{CONSIDERAÇÕES FINAIS}

Em síntese, os resultados desta investigação mostraram que a produção científica na área de enfermagem em cuidados intensivos, no Brasil, encontrase ainda incipiente, não se observando crescimento no decorrer das últimas décadas. Além disso, outra lacuna observada foi a ausência de ensaios clínicos que dêem sustentação para a prática assistencial de enfermagem, apontando, portanto, caminhos a serem buscados pelos enfermeiros intensivistas brasileiros.

A falta de estudos internacionais que permitissem uma análise mais abrangente dos resultados encontrados nesta investigação e a inclusão de apenas dez periódicos nacionais de enfermagem são limitações deste estudo. No entanto, a continuidade dada a estudos da mesma natureza, realizados em duas décadas anteriores, trouxeram resultados que permitiram verificar a evolução da produção científica de enfermagem no Brasil, na área de cuidados intensivos. Mais do que mostrar o passado e o estado atual das investigações, os resultados trazem subsídios para a reflexão das grandes necessidades de investigação ainda existentes nessa área, após mais de trinta anos de enfermagem em UTI, no Brasil.

\section{REFERÊNCIAS}

1. Padilha KG, Pierin AM, Ide CA, Braz E, Farias GM, Takahashi OC. Pesquisa em enfermagem médico-cirúrgica no Brasil. Rev Paul Enfermagem. 1983; 3(5): 167-9.

2. Koizumi MS. Perfil da pesquisa de enfermagem em terapia intensiva no Brasil. Rev Esc Enfermagem USP. 1997; 31(3): 468-85.

3. Koizumi MS, Miyadahira AMK, Takahashi EIU. Análise retrospectiva das pesquisas de enfermagem em terapia intensiva: 1975-1984. Rev Esc Enfermagem USP. 1986; 20(1): 5-17.

4. Padilha KG, Kimura M, Miyadahira AMK. Produção científica de enfermagem em terapia intensiva no Brasil: análise retrospectiva de 1985 a 1994. In: Encontro de Enfermagem E Tecnologia, 5. Programa São Paulo: Centro de Estudos de Enfermagem Oito de Agosto; 1996. p. 384-8.

5. Coordenação de Aperfeiçoamento de Pessoal de Nível Superior (CAPES). Apresenta relação de periódicos e 
classificação relativa a dados de 2003. Brasilia (DF): Ministério da Educação; c2003. [citado 2005 Jul 11]. Disponível em: http://www.capes.gov.br

6. Adami NP, Salzano SDT, Castro RAP, Stefanelli MC. Situação da pesquisa em enfermagem em hospitais do município de São Paulo. Rev Latinoam Enfermagem. 1996; 4(1): 5-20.

7. Cruz ICF, Sobral VRS, Gauthier J. Produção e consumo científico-artístico: a dialética do desenvolvimento profissional de enfermagem. Texto \& Contexto Enfermagem. 1996; 5(1): 82-91.
8. Azevedo NDA. Formação do enfermeiro especialista em terapia intensiva. Rev Baiana Enfermagem. 1995; 8(1/2): 160-8.

9. Fernandes JD, Guimarães A, Araújo FA, Reis LS, Gusmão MCCM, Trabuco MQB, et al. Construção do conhecimento de enfermagem em unidades de tratamento intensivo: contribuição de um curso de especialização. Acta Paul Enfermagem. 2004; 17(3): 325-32.

10. Correa AK. Pesquisas de enfermagem em terapia intensiva: análise de dissertações e teses. Rev Esc Enfermagem USP. 2000; 34(3): 252-7. 